\title{
60 años de cirugía de la válvula mitral. U na historia de exploradores, pioneros, héroes y conquistadores de nuestros tiempos
}

\section{Dr. Ricardo Zalaquett S. \\ Sixty years of mitral valve surgery}

\begin{abstract}
On June 10, 1948, Charles Bailey, MD, operated successfully a mitral valve stenosis in Philadelphia and six days later, Dwight Harken, MD, performed the same operation in Boston, marking the onset of cardiovascular surgery. These successful operations were preceded by several failures and even deaths, that had to be overcome by both pioneers. This manuscript reviews several cases and situations that these surgeons had to face during the development of cardiovascular surgery, that changed the natural history of cardiac diseases. The history culminates with the successful mitral valve replacement, performed by Albert Starr using a mechanical valve designed by him and Lowell Edwards, a retired engineer whose primary interest was to develop an artificial heart. The first mitral commissurotomy in Chile was performed by Svante Törnvall, MD, and Pedro Uribe, MD, at van Buren Hospital in Valparaiso in December, 1950. The first mitral valve replacement was performed by Hugo Salvestrini, MD, in 1964 at the Catholic University Hospital (Rev Méd Chile 2009; 137: 1253-60).
\end{abstract}

(Key w ords: Cardiovascular surgical procedures; History of Medicine; Mitral valve)

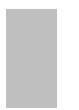

Recibido el 30 de junio, 2008. Aceptado el 9 de enero, 2009.

Departamento de Enfermedades Cardiovasculares, Facultad de Medicina, Pontificia Universidad Católica de Chile. Santiago de Chile.

$\mathrm{E}$ 10 de junio de 1948 Charles P. Bailey operó por primera vez con éxito una estenosis de la válvula mitral, en la ciudad de Filadelfia. Seis días más tarde, el 16 de junio del mismo año, Dwight E. Harken, en Boston, operó otro caso exitoso de estenosis mitral. Con estas 2 intervenciones pioneras se inició la cirugía de corazón propiamente tal,

$\overline{\text { Correspondencia a: Dr. Ricardo Zalaquett S. Hospital Clíni- }}$ co Pontificia Universidad Católica de Chile. Marcoleta 367, $6^{\circ}$ piso. Teléfonos: 6333030 - 3543231. Fax: 6390108.

E mail: rzalaque@med.puc.cl ya que si bien en 1896 Ludwig Rehn ${ }^{1}$, en Francfort, había suturado una herida de corazón, Robert Gross, en Boston, en 1938 había ligado un ductus arterioso persistente; Clarens Crafoord, en Estocolmo, en 1944, había operado una coartación aórtica, y, más tarde, en 1945, Alfred Blalock, en Baltimore, había efectuado la anastomosis de una rama del cayado aórtico a la arteria pulmonar en un paciente con una tetralogía de Fallot, la cirugía cardiovascular era, en la práctica, hasta 1948, una cirugía "extracardiaca" 2 .

Para tener una idea de lo que hasta ese entonces significaba ser portador de una valvulopatía mitral es oportuno releer lo que José Luis Arraño, interno del Hospital San Juan de Dios a 
comienzos de los años 30, en un librillo titulado "Morbus", publicado en 1934, escribió acerca de un paciente portador de una valvulopatía mitral ${ }^{3}$.

\section{UN MITRÁLICO}

Ya ni el mismo se acuerda de aquella tarde, en que vino un cortejo de amigos y parientes a dejarlo.

Después que se acostó, entre pestañeos rápidos y tragos costosos de saliva, se fueron despidiendo con palabras de aliento y resignación para él. Su esposa le dejó un beso en la boca y fue la última en irse.

El médico le diagnosticó una insuficiencia mitral y disertó muchas veces elocuentemente al pie de la cama, sobre las secuelas que deja el reuma en el corazón. Al comienzo él escuchaba atentamente las lecciones, pero después...

Al domingo siguiente, vinieron a visitarlo su madre y esposa. Traían un saludo en los labios y un paquete en los brazos. Al siguiente, solo su esposa, alcanzó ya sin paquetes. De vez en cuando, un amigo aparecía en la puerta de la sala buscándolo, escudriñador, entre los demás.

Un día se compensó y pidió el alta...

Trato de recomenzar pero se descompensó y una tarde volvió a la sala, esta vez sin cortejo a ocupar otro número.

De esto hace varios años. Ya ni el mismo se acuerda.

Ahora es el paciente crónico, el decano de los enfermos. Y comprende que es sólo un capítulo de la patología, que se mantiene a fuerzas de cucharadas y gotas. La digitalina es su razón de ser; y a un hombre que tiene tal deuda con un remedio no puede quedarle hueco en el corazón, cuanto menos si está enfermo; por eso no ambiciona, no pide nada, no fragua un plan para el futuro, se siente incapaz de engendrar derechos: todo lo encuentra bueno, hasta la comida del hospital. Y es que en cada poción, ha ingerido una gran dosis de estoicismo.

No tiene nada de qué preocuparse, y en momentos de optimismo se ha creído una flor delicada mantenida en un invernadero.

Ha oído muchas veces a la banda de músicos recorrer los patios el día del Hospital, se sabe al dedillo el reglamento de la sala, conoce a todo el personal de la clínica, es el único que duerme cuando alguien agoniza o se queja en la noche, el que les escribe las cartas a los analfabetos o a los que están graves, y podría dar una interesante conferencia sobre insuficiencia cardíaca.

Es el repitente de la sala.

Sus amigos son los enfermos del lado, que se suceden con una modalidad cinematográfica, le cuentan sus aventuras y un buen día se van de alta. No conoce más del mundo actual, que empieza más allá de los bordes de su catre, que lo que le llega por boca de ellos, pues es el único enfermo al que jamás el suplementero le ofrece el diario.

¿Y él no tiene nada que contar?

El primer capítulo de su vida, lo resumió el interno en una historia de achaques; el segundo, lo está escribiendo el practicante todas las mañanas, es un folletín morboso en el que están inscritos el pulso, la temperatura, la presión, los específicos, en fin, la evolución de su insuficiencia.

Y él, como todos los enfermos guarda esos papeles en el respaldo del catre, pues, bien puede encarpetar la vida a sus espaldas quien la mira a través de la portería del Hospital.

A partir de junio de 1948 los pacientes "mitrálicos" dejarían de "encarpetar la vida a sus espaldas y de mirarla a través de la portería del Hospital".

Pero, antes de Bailey y Harken hubo otros cirujanos que concibieron procedimientos para tratar la estenosis mitral, otros llevaron a cabo estos procedimientos sin éxito, y otros, finalmente, a pesar de efectuar una operación exitosa, fueron completamente ignorados, no pudiendo repetir la intervención. Al respecto es necesario recordar primero a Sir Lauder Brunton, distinguido cardiólogo de Londres, quien publicó lo que él llamó una nota preliminar en Lancet, con el inequívoco título "Preliminary note on the possibility of treating mitral stenosis by surgical methods", en $1902^{4}$. Uno de sus colegas escribió en la edición siguiente del Lancet lo siguiente: "es posible hacer muchas cosas que son inútiles y algunas que son dañinas" 5 .

Más tarde, en 1923, Elliot Cuttler, apoyado por su cardiólogo, el famoso Samuel Levine, en el Hospital Peter Bent Brigham, en Boston y luego de un arduo trabajo de cirugía experimental en perros y gatos, efectuó una "valvotomía mitral 
transventricular". La descripción de Cuttler de esta operación es casi escalofriante: "... el cuchillo, un cuchillo de amígdalas ligeramente curvo, fue empujado hasta encontrar lo que nos pareció debía ser el orificio mitral... se efectuó un corte en lo que pensamos era el velo aórtico de la válvula mitral... el cuchillo fue rápidamente girado y se efectuó otro corte en el lado opuesto de la apertura...”. La paciente, una niña de 11 años, sobrevivió por varios años, pero los 6 intentos ulteriores de Cuttler fracasaron, falleciendo los pacientes por regurgitación mitral masiva. En 1928 Cuttler, desilusionado, abandonó completamente la valvotomía mitral transventricular ${ }^{7}$.

En el medio oeste norteamericano, Allen y Graham, por su parte, desarrollaron un "cardioscopio" para visualizar la válvula mitral el que era introducido a través de la aurícula izquierda. En noviembre de 1923 operaron con este cardioscopio a una mujer de 31 años, pero la paciente falleció en la mesa de operaciones, lo que fue atribuido a la anestesia ${ }^{8}$. En 1925, en Londres, prácticamente un cuarto de siglo antes que Bailey y Harken, Sir Henry Souttar operó a una mujer de 19 años portadora de una estenosis mitral. El procedimiento en este caso consistió en una dilatación digital de la válvula mitral, introduciendo el dedo índice a través de una incisión en la orejuela izquierda? 9 . El dedo de Souttar habría permanecido en la aurícula izquierda por uno o dos minutos. La paciente sobrevivió la operación y vivió 5 años más, falleciendo finalmente por una embolia cerebral. A pesar de su éxito, Souttar no volvió a operar otro caso de estenosis mitral. De acuerdo con Andreas Naef, Souttar, en una carta dirigida a Harken 25 años más tarde, respondiendo a las razones de por qué no había vuelto a operar a otro paciente, señaló: "porque no pude obtener otro caso... en ese entonces era un acto de fe entre los internistas, de que las válvulas no eran importantes y que la única cosa que importaba era la condición del músculo cardiaco"10.

Al final de los años veinte del siglo recién pasado, 10 pacientes mitrálicos habían sido operados y sólo 2 de ellos habían sobrevivido la operación, ninguno de los cuales habría experimentado una mejoría objetiva. Descorazonados por estos malos resultados los cirujanos abandonaron la válvula mitral como un objetivo quirúrgi$\mathrm{Co}^{11}$. Así, tuvieron que pasar más de 20 años para que Charles Bailey (Figura 1), en Filadelfia, el 10 de junio de 1948, operara por primera vez con éxito una estenosis mitral en una joven mujer de 24 años, la que sobrevivió a una incisión comisural de su válvula mitral con un bisturí digital, introduciendo Bailey el término "comisurotomía", para ser seguido 6 días después, en Boston, por

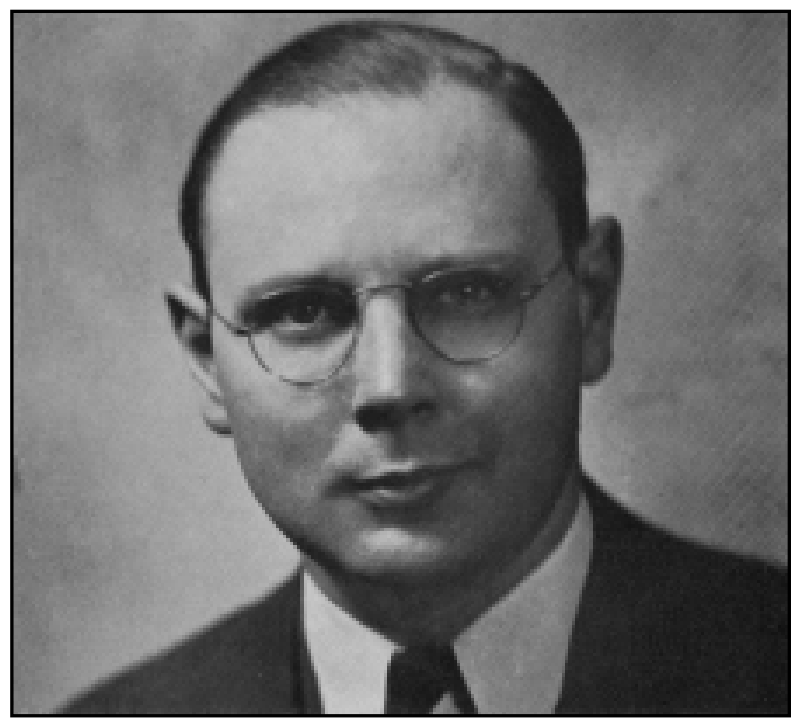

Figura 1. Fotografía de la época de Charles Bailey, cirujano que el 10 de junio de 1948 operó por primera vez con éxito una estenosis mitral en la cuidad de Filadelfia, introduciendo el término "comisurotomía". 
Dwight Harken (Figura 2) ${ }^{12,13}$. Éste, por su parte, efectuó una escisión de una porción de la comisura anterolateral de la válvula mitral de una mujer de 27 años, con un instrumento parecido al usado por Cuttler, aceptando, como él mismo señaló, un cierto grado de regurgitación e introduciendo el término de "valvuloplastía"13. En ambos casos, el acceso a la válvula mitral fue a través de la orejuela izquierda, por una toracotomía posterolateral izquierda y pericardiotomía longitudinal. Con la comisurotomía y la valvuloplastía mitral cerrada se había iniciado la era del tratamiento quirúrgico propiamente tal de las patologías cardiacas adquiridas, para ser seguido luego por las malformaciones congénitas del corazón, todo lo cual significó una revolución en el tratamiento de las enfermedades cardiovasculares. Estas operaciones habían llegado para quedarse y ser perfeccionadas. Bailey y Harken habían escrito para siempre su nombre en la historia de la cardiología y cirugía cardiovascular.

Pero, el camino de estos grandes pioneros había sido duro. Harken había perdido un paciente en marzo de 1947 y Bailey uno en 1945 y otro en 1946, y probablemente otro más en marzo de 1948. El caso de Bailey es especialmente sorprendente. Como relata Andreas Naef en su "The story of thoracic surgery", éste literalmente había sido condenado al ostracismo por los círculos médicos de Filadelfia y había perdido incluso sus privilegios operatorios en sus hospitales habituales ${ }^{10}$. A diferencia de Harken, su actividad quirúrgica no estaba protegida por un ambiente académico. A pesar de todo esto, Bailey estaba completamente convencido de estar en el camino correcto y poseía una personalidad a toda prueba, capaz de enfrentar las más grandes de las adversidades. De hecho, el mismo 10 de junio de 1948, Bailey había programado 2 casos de estenosis mitral en 2 hospitales diferentes. El paciente de la mañana falleció durante la operación. Este era un caso extremo ya que se trataba de un paciente sumamente grave. Sin embargo, Bailey no se amilanó y en la tarde del mismo día, y como él mismo habría relatado, "nos juntamos todo el frustrado equipo y manejamos rápidamente al hospital episcopal para comenzar la otra operación, antes de que las malas noticias de la mañana pudieran hacer que los administradores del hospital nos prohibieran hacer la operación"10,11.

Como ha quedado registrado en la historia de la cirugía, este segundo caso de la tarde del 10 de junio de 1948 fue un éxito y la paciente tuvo un postoperatorio sin incidentes. Tanto así que, una semana después de la operación, Bailey viajó presuroso con su paciente de Filadelfia a Chicago, donde éste presentó el caso en el congreso del American College of Chest Physicians ${ }^{10}$. Por su

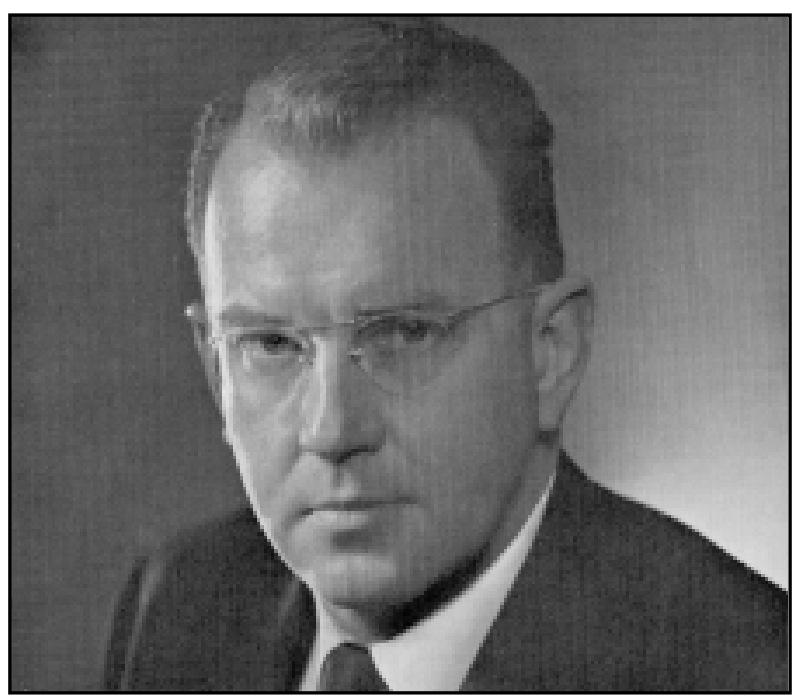

Figura 2. Fotografía de la época de Dwight Harken, cirujano que el 16 de junio de 1948 operó el segundo caso de estenosis mitral con éxito, en la ciudad de Boston, introduciendo el término "valvuloplastía". 
parte, Harken conocía muy bien a Joe Garland, el entonces editor del New England Journal of Medicine, por lo que pudo publicar su caso ese mismo año, a diferencia de Bailey que no pudo publicar hasta el año siguiente ${ }^{12,13}$. Desde entonces ha persistido la fatua discusión de si la prioridad en la cirugía mitral y, por ende, de la cirugía de corazón, corresponde a quien operó efectivamente el primer caso o a quien efectuó la primera publicación. Tres meses más tarde, en septiembre de 1948, Russell Brock, en Londres, efectuó también una valvulotomía mitral exitosa. Brock había efectuado ya una valvulotomía pulmonar en una tetralogía de Fallot en febrero de ese año, operación por la que es recordado en la historia de la cirugía cardiovascular, convirtiéndose luego en "Lord Brock"10,11.

Sin embargo, como a menudo sucede, hubo un cuarto cirujano, quien efectuó realmente la primera cirugía exitosa para una estenosis mitral propiamente tal de ese año 1948. Este fue Horace Smithy, en Charleston, Carolina del Sur, utilizando la antigua técnica de Cuttler. Smithy operó su primer paciente el 10 de enero de 1948, es decir 5 meses antes que Bailey y Harken. A diferencia de Cuttler, no usó un acceso transventricular izquierdo a la válvula mitral sino que a través de la aurícula izquierda, pero sí siguió el concepto de éste de "valvulectomía parcial", utilizando un instrumento parecido a un biótomo. Smithy operó 7 pacientes de los cuales 6 sobrevivieron. Smithy no sólo ha sido olvidado porque la técnica de la valvulectomía resultó ser la menos apropiada, sino porque murió de una estenosis aórtica ese mismo año, no pudiendo publicitar su trabajo, como lo hicieron Bailey y Harken ${ }^{10,11}$.

De estos 4 pioneros fue Bailey quien desarrolló la técnica más apropiada muy tempranamente, conceptuando el procedimiento de "comisurotomía mitral cerrada", que se mantendría por muchos años y que es la base de la actual comisurotomía mitral percutánea con balón al percatarse, en uno de sus primeros casos, que eran las comisuras las que se abrían con su dedo, independientemente de la fuerza que se aplicara ${ }^{12,14}$. Esto rápidamente originó la idea, ya insinuada por Cuttler en 1929, de usar "dilatadores mecánicos expandibles", el primero de los cuales fue diseñado por Charles Dubost en París, el que era introducido en forma ciega a través de la orejuela izquierda. Entre 1953, año en que introdujo su dilatador, y 1962, Dubost operó 965 pacientes, con una mortalidad de sólo 2\%. A pesar de esto, el dilatador transatrial no logró una mayor aceptación ${ }^{11,15}$. La técnica de Dubost fue rápidamente mejorada por Logan y Tubss, los cuales diseñaron un instrumento similar, pero que era ahora introducido a través del ápex del ventrículo izquierdo por una toracotomía anterolateral izquierda, controlando la posición transmitral y la magnitud de la dilatación de la válvula mitral por palpación digital transauricular (el dedo índice era introducido a través de una jareta en la orejuela izquierda) ${ }^{16}$. Es interesante señalar que este dilatador mitral ajustable concebido por Oswald Tubss, a partir del dilatador rígido utilizado por Andrew Logan, de Edimburgo, fue fabricado por la "Genito-Urinary Manufacturing Company", la que lo vendió en gran cantidad en todo el mundo, sin pagar derechos a quienes lo idearon, ya que éstos no lo patentaron ${ }^{10}$.

Con el desarrollo de la cirugía con circulación extracorpórea a comienzo de los años cincuenta, por John Gibbon ${ }^{17}$ y Walton Lillehei ${ }^{18}$, la cirugía mitral cerrada dio paso a la valvuloplastia mitral abierta, inicialmente a través de una toracotomía derecha con canulación de la arteria femoral y luego a través de una esternotomía media, para llegar finalmente al primer reemplazo valvular protésico. Éste se efectuó el 25 de agosto de 1960 por Albert Starr, en la Universidad de Oregón, utilizando una prótesis de canastillo y bola diseñada por él, en conjunto con Lowell Edwards, ingeniero jubilado y con un Parkinson inicial, que buscaba en qué ocupar su tiempo y a quien el joven cirujano que era Starr convenció de abandonar su idea original de desarrollar un corazón mecánico por el de una prótesis valvular (Figura $3)^{19,20}$. Esta primera paciente operada por Starr falleció súbitamente la noche de su operación, luego de haber despertado de la anestesia, al ser colocada en decúbito lateral derecho. Al revisar la radiografía de tórax postoperatoria, Starr y sus cols cayeron en la cuenta que lo que habían interpretado como un neumotórax era en realidad una burbuja de aire en la aurícula izquierda. La paciente había fallecido de una embolia aérea ${ }^{20}$.

El 21 de septiembre de 1960, Albert Starr operó ahora a un hombre de 52 años con una secuela reumática, quien había sido previamente sometido a 2 comisurotomías mitrales cerradas, cambiando su válvula mitral enferma por la prótesis de canastillo y bola, que pasó a conocerse 
desde entonces como prótesis valvular "StarrEdwards". La operación fue todo un éxito y el paciente vivió por 10 años una vida normal, hasta que murió al caerse de una escalera (Figura 4$)^{20}$. El recambio valvular mitral se convirtió en un procedimiento frecuente, pero con una mortalidad inicial de 50\%, si bien ésta cayó a menos de 5\% en la experiencia de Starr en los próximos 6 años ${ }^{20}$.

Desde sus trabajos en animales, Starr y Edwards estaban concientes de la trombogenicidad de estas prótesis valvulares mecánicas, por lo que desde el primer paciente que sobrevivió todos fueron anticoagulados de por vida con cumadina, originalmente un veneno para ratas, lo que a su vez era un problema en sí mismo ${ }^{20}$. Es por esto que Alain Carpentier (Figura 5), en París, estimulado por la dolorosa experiencia de un joven artista limitado de por vida por una embolia cerebral originada en una prótesis mecánica, desarrolló, finalmente, luego de explorar diferentes alternativas, una "bioprótesis" a partir de una válvula aórtica de cerdo tratada con glutaraldehido, sustancia usada en la curtiembre de cuero, que no va a requerir de anticoagulación, pero que va a tener una durabilidad más limitada ${ }^{21}$. La primera bioprótesis se implantó en Paris en $1967^{22}$. Pero, sorprendentemente, en contra de sus propios intereses financieros, Carpentier consideró que la válvula mitral nativa era superior a la mejor de las prótesis, introduciendo una serie de procedimientos quirúrgicos para preservar la válvula mitral, basados en principios funcionales, anatomopatológicos y etiológicos de la valvulopatía mitral ${ }^{23}$.

En nuestro país, de acuerdo al doctor Adolfo Reccius, autor del libro "Historia y Desarrollo de la Cirugía Torácica en Chile", la primera comunicación sobre el tratamiento quirúrgico de la estenosis mitral fue hecha por la Unidad Médico Quirúrgica Cardiovascular del Hospital Carlos Van Buren de Valparaíso ${ }^{24}$. Siempre de acuerdo con éste, la primera comisurotomía mitral cerrada fue efectuada en Chile por los cirujanos de dicha unidad Svante Törnvall y Pedro Uribe, en diciembre de 1950, dos años y medio después de los primeros casos efectuados por Bailey y Harken en Estados Unidos de Norteamérica y antes que en muchos otros centros cardiovasculares impor-

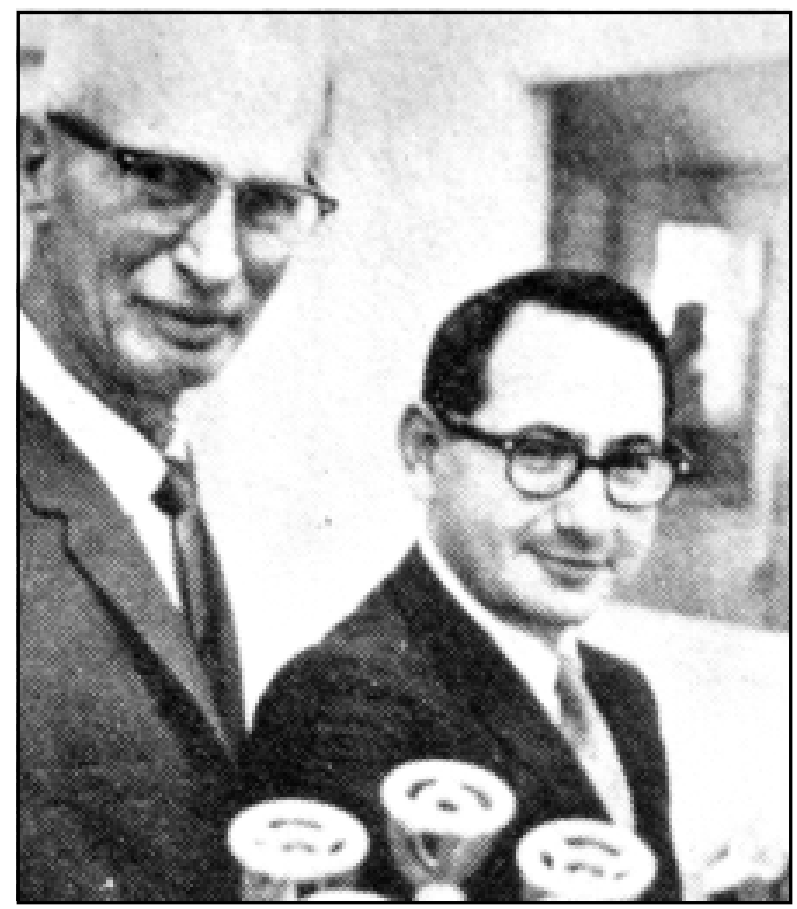

Figura 3. Lowell Edwards (izquierda) y Albert Starr (derecha), ingeniero y cirujano, creadores de la primera prótesis valvular cardiaca implantada exitosamente en posición mitral, el 21 de septiembre de 1960, en la ciudad de Oregón (Gentileza de Edwards Lifesciences).

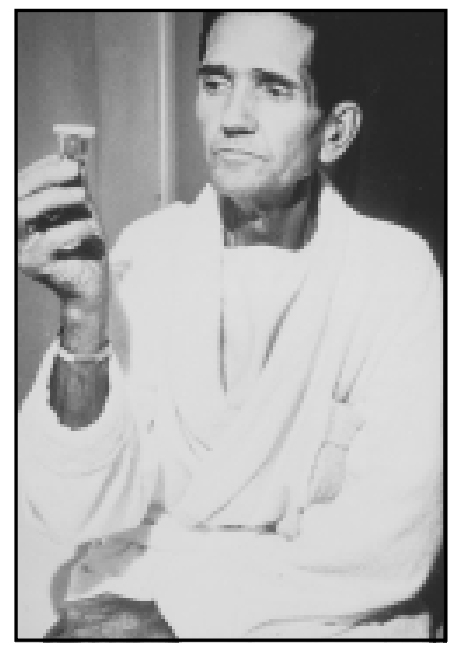

Figura 4. Fotografía del primer paciente operado exitosamente por una estenosis mitral por Albert Starr, cuya válvula fue reemplazada por una prótesis de "canastillo y bola" diseñada por éste y Lowell Edwards, un modelo de la cual sostiene el paciente con su mano derecha (Gentileza de Edwards Lifesciences). 


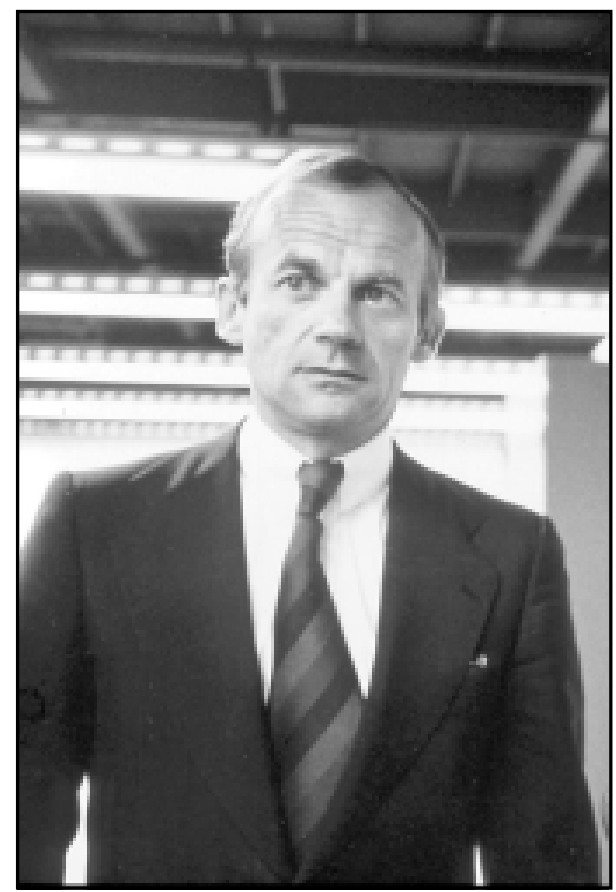

Figura 5. Fotografía de la época de Alain Carpentier, inventor de las "bioprótesis valvulares cardiacas", la primera de las cuales fue implantada por él, en 1967, en París (Gentileza de Edwards Lifesciences).

tantes del mundo (Figura 6). En el Hospital Clínico de la Universidad Católica la primera comisurotomía mitral cerrada se efectuó el 17 de marzo de 1953, por los cirujanos Lucchini, Rencoret y Dubernet. Sin embargo, casi todas las comisurotomías mitrales subsiguientes efectuadas en nuestro hospital, por un largo periodo de tiempo, tuvieron como cirujano al doctor Hugo Salvestrini, ayudado siempre por los doctores Lucchini y Dubernet. El 15 de mayo de 1964 se llevó a cabo en el Hospital de la Universidad Católica, por este mismo equipo quirúrgico, al que se agregó ahora el doctor Gustavo Maturana, y con la colaboración del cirujano norteamericano Peter Gall, de visita en Chile, el primer reemplazo de una válvula mitral con una prótesis de Starr-Edwards, de Chile y muy probablemente de Sudamérica ${ }^{25}$ (Figura 7).

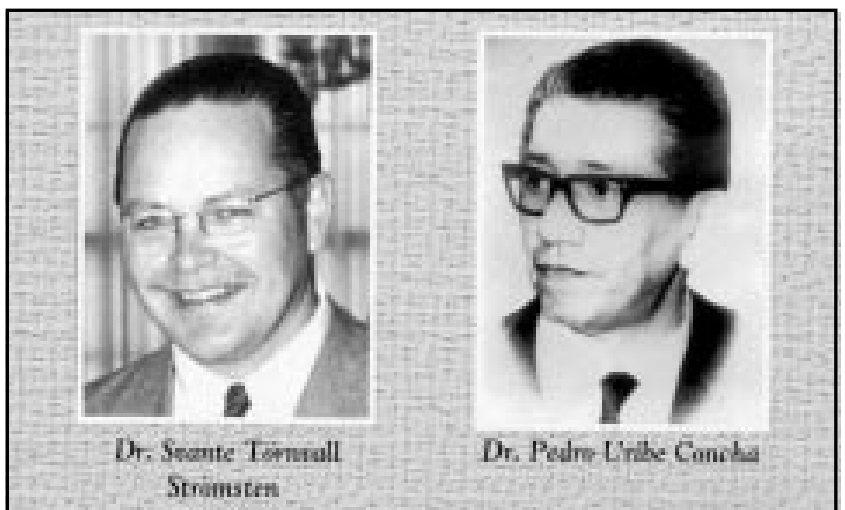

Figura 6. Los cirujanos Svante Törnvall y Pedro Uribe, de la Unidad Médico Quirúrgica Cardiovascular del Hospital Carlos van Buren de Valparaíso, los que efectuaron la primera comisurotomía mitral cerrada en Chile, en diciembre de 1950. (Gentileza del doctor Lorenzo Cubillos).

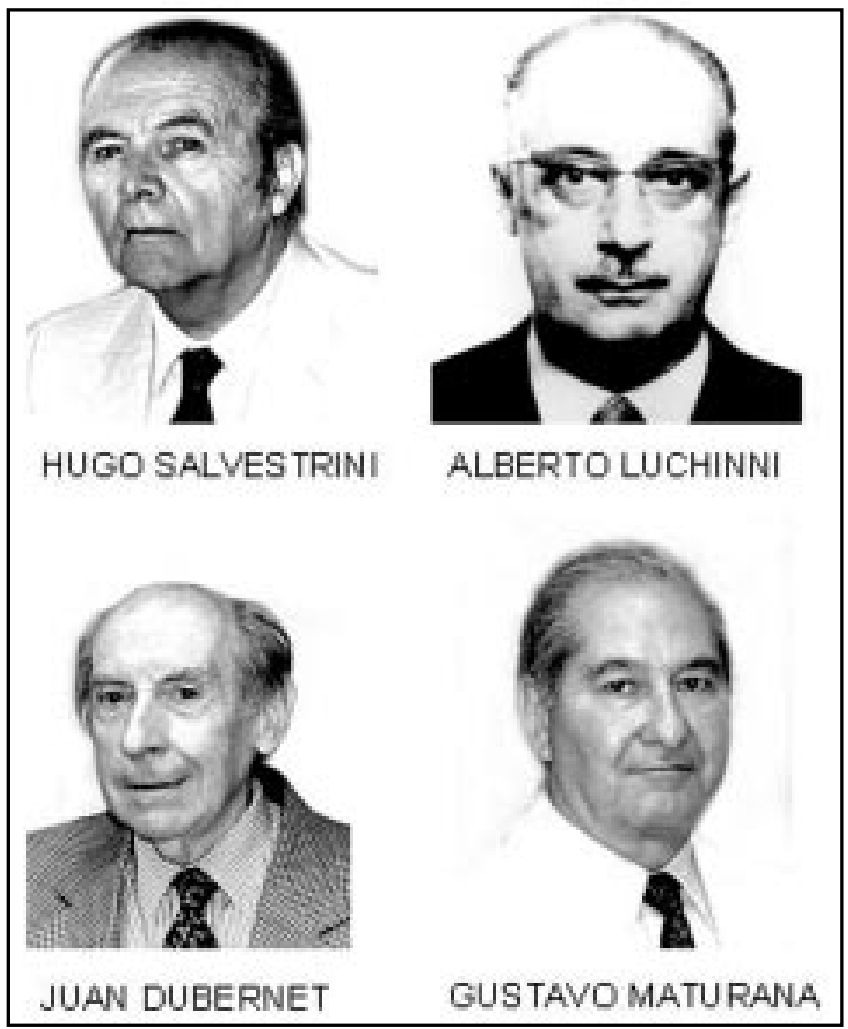

Figura 7. Equipo quirúrgico del Hospital Clínico de la Pontificia Universidad Católica que efectuó el primer reemplazo valvular mitral en Chile y, muy probablemente, en Sudamérica, el 15 de mayo de 1964. 


\section{REFERENCIAS}

1. Zalaquett R. 1896-1996. Cien años de Cirugía Cardiaca: Ludwig Rehn y la primera operación al corazón. Rev Méd Chile 1996; 124: 1281-4.

2. Zalaquett R. Y de cómo se llegó finalmente a la cirugía a corazón abierto. Parte I. Rev Chil Cardiol 2000; 19: 97-103.

3. Arraño JL. Morbus. Santiago, Chile: Julio Walton editor, 1934.

4. Brunton L, Edin MD. Preliminary Note on the Possibility of Treating Mitral Stenosis by Surgical Methods. Lancet 1902; 1: 352.

5. FisHer T. Letter to the editors. Lancet 1902; 1: 547-8.

6. Cutler EC, Levine SA. Cardiotomy and Valvulotomy for Mitral Stenosis: Experimental Observations and Clinical Notes Concerning an Operated Case with Recovery. Boston Med Surg J 1923; 188: 1023-7.

7. Cutler EC, Веск CS. The Present Status of the Surgical Procedures in Chronic Valvular Disease of the Heart: Final Report of all Surgical Cases. Arch Surg 1929; 18: 403-16.

8. Allen DS, Graham EA. Intracardiac Surgery - A New Method: Preliminary Report. Jama 1922; 79: 1028-30.

9. SoutTAR HS. The Surgical Treatment of Mitral Stenosis. Br Med J 1925; 2: 603-6.

10. NAEF AP. The Story of Thoracic Surgery. Germany: Hans Huber Publishers, 1990.

11. ElLis FH Jr. Surgery for Acquired Mitral Valve Disease. Philadelphia \& London: W.B. Saunders Company, 1967.

12. Bailey CP. The Surgical Treatment of Mitral Stenosis (Mitral Commisurotomy). Dis Chest 1949; 15: 377-93.

13. Harken DE, Ellis LB, Ware PF, Norman LR. The Surgical Treatment of Mitral Stenosis. N Engl J Med 1948; 239: 801-9.
14. Bailey CP, Glover RP, O'Neill TJ. The Surgery of Mitral Stenosis. J Thoracic Surg 1950; 19: 16-45.

15. Dubost C, Blondeau P, Pinwica A. Instrumental Dilatation Using the Transatrial Approach in the Treatment of Mitral Stenosis: A Survey of 1000 Cases. J Thoracic Cardiovasc Surg 1962; 44: 392-407.

16. Logan A, Turner R. Surgical Treatment of Mitral Stenosis with Particular Reference to the Transventricular Approach with a Mechanical Dilator. Lancet 1959; 2: 874-80.

17. Zalaquett R. Cincuentenario de la Máquina CorazónPulmón. Rev Méd Chile 2003; 131: 1337-44.

18. Zalaquett R. C. Walton Lillehei, MD, PHD (19181999): Héroe y Pionero de la Cirugía a Corazón Abierto. Rev Chil Cardiol 1999, 18: 95-104.

19. STARr A, Edwards ML. Mitral Replacement: Clinical Experience with a Ball-Valve Prosthesis. Ann Surg 1961; 154: 726-40.

20. Starr A. The Artificial Heart Valve. Nature Medicine 2007; 13: 1160-4.

21. Carpentier A. The Surprising Rise of Nonthrombogenic Valvular Surgery. Nature Medicine 2007; 13: 1165-8.

22. Carpentier A, Blondeau P, Laurens B, Hay A, Laurent D, Dubost C. Mitral and Tricuspid Valve Replacement with Frame-Mounted Aortic Heterografts. J Thoracic Cardiovasc Surg 1968; 56: 388-94.

23. Carpentier A. Cardiac Valve Surgery - the "French Correction". J Thoracic Cardiovasc Surg 1983; 86: 232-7.

24. Reccius A. Historia y Desarrollo de la Cirugía Torácica en Chile. Valparaíso: Imprenta y Litografía Universo, S.A., 1957.

25. Salvestrini H, Dubernet J, Valdivieso J, Lucchini A, Maturana G, Wilson C et al. Reemplazo de la válvula mitral con circulación extracorpórea. Arch Soc Cir Chile 1964; 16: 533-9. 